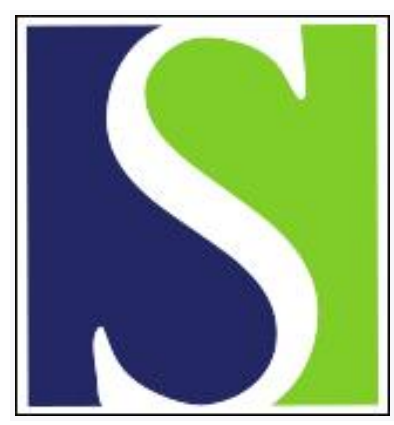

Scand J Work Environ Health 1996;22(5):360-363

https://doi.org/10.5271/sjweh.154

Issue date: Oct 1996

Glutathione S-transferase M1 null genotype as a risk modifier for solvent-induced chronic toxic encephalopathy

by Söderkvist P, Ahmadi A, Åkerbäck A, Axelson O, Flodin U

Key terms: chronic toxic encephalopathy; molecular epidemiology; polymorphism; solvent exposure

This article in PubMed: www.ncbi.nlm.nih.gov/pubmed/8923609

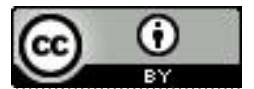




\title{
Glutathione S-transferase M1 null genotype as a risk modifier for solvent-induced chronic toxic encephalopathy
}

\author{
by Peter Söderkvist, PhD, ${ }^{1}$ Ahmad Ahmadi, BSc, ${ }^{1}$ Anita Åkerbäck, BSc, ${ }^{1}$ Olav Axelson, MD, ${ }^{1}$ \\ Ulf Flodin, $M D^{1}$
}

\begin{abstract}
Söderkvist P, Ahmadi A, Åkerbäck A, Axelson O, Flodin U. Glutathione S-transferase M1 null genotype as a risk modifier for solvent-induced chronic toxic encephalopathy. Scand J Work Environ Health 1996:22:360_70.
\end{abstract}

\begin{abstract}
Objectives Exposure to organic solvents increases the risk of neuropsychiatric disability or chronic toxic encephalopathy (CTE). Polymorphisms in the biotransformation of xenobiotics and solvents may influence individual susceptibility to develop toxic effects. In this study the problem of whether there could be any association between the glutathione S-transferase M1 (GSTM1) null genotype and the risk for CTE, with regard to solvent exposure, was investigated.

Methods Sixty patients referred to a clinic because of some degree of psychiatric or neurological symptoms, as well as exposure to solvents, were examined by means of a validated questionnaire and psychometric testing. The degree of exposure to solvents was assessed by a thorough interview. According to clinical findings, the patients were classified into three categories as those with solvent-induced CTE, those with incipient CTE, and those who were non-CTE patients. Afterwards, leukocyte DNA (deoxyribonucleic acid) was isolated and the GSTM1 null genotype was determined by an assay based on polymerase chain reaction, blindly with regard to both exposure and disease status.

Results The relative proportion (RP) of GSTM1 null genotypes was significantly increased for patients with a diagnosed CTE when they were compared with non-CTE patients (RP 2.55, 95\% confidence interval 1.0-6.2). Dichotomizing the patients by high and low exposure revealed an increased risk for both GSTM1 gene carriers and the GSTM1 null genotype in the high-exposure group, the relative risks (RR) being 4.5 and 7.9 , respectively. The chi-square for the Mantel extension for trend was $6.2(\mathrm{P}=0.025)$.

Conclusions The GSTM1 null genotype acts as a risk modifier for CTE among patients occupationally exposed to solvents. The risk seems to increase in a dose-dependent fashion.
\end{abstract}

Key terms chronic toxic encephalopathy, molecular epidemiology, polymorphism, solvent exposure.

Exposure to organic solvents is common in industrial and other work tasks and an increased risk of neuropsychiatric disability or chronic toxic encephalopathy, sometimes called psychoorganic syndrome, has been found in several epidemiologic studies in the Nordic and other industrialized countries (1). The mechanism of action for chronic toxic encephalopathy induced by solvents is unclear, and hypotheses of the metabolic activation of solvents to toxic intermediates have been suggested. Most xenobiotics and the majority of solvents are biotransformed by enzymes through oxidative or reductive reactions, usually followed by conjugation with some endogenous substrate (2). Polymorphic expressions of the enzymes involved may play a role in interindividual susceptibility to toxic agents. Genetically based meta- bolic susceptibility has been considered particularly for carcinogens. Glutathione S-transferases (GST) play a central role in inactivating a large number of reactive intermediates after the addition of reduced glutathione and after subsequent enzymatic conversions form corresponding mercapturic acid. So far, four classes of soluble glutathione $S$-transferases have been identified, referred to as the $\alpha, \mu, \Pi$ and $\tau$ glutathione $S$-transferases (3). The most widely expressed $\mu$ gene, GSTM1, is polymorphic, and four common phenotypes result from homo- and heterozygous combinations of GSTM $1 * 0$, GSTM $1 *$ A and GSTM1*B gene products. Among $40-60 \%$ of the Caucasian population is homozygous for the deleted GSTM $1 * 0$ allele (hereafter termed GSTM1-), and they express no GSTM1 enzyme (4). In the Swedish popula-

1 Department of Occupational and Environmental Medicine, Faculty of Health Sciences, University Hospital, Linköping, Sweden.

Reprint requests to: Dr Peter Söderkvist, Department of Cellbiology, Faculty of Health Sciences, University Hospital, S-581 85 Linköping, Sweden. E-mail: petso@ymk.liu.se. 
tion the frequency for GSTM1- is approximately 53\% (personal communication, Dr Agneta Rannug, Department of Biochemical Toxicology, National Board for Occupational Safety and Health, S-17184, Solna, Sweden). Impaired ability to detoxify electrophiles may increase the susceptibility to chemically-induced toxicity and influence the development of chronic toxic encephalopathy. Therefore, we found it interesting to assess the GSTM1 null genotype among our patients with occupational exposure to solvents.

\section{Subjects and methods}

The study population comprised 60 patients who visited the Department of Occupational and Environmental Medicine, at the University Hospital in Linköping, from January 1992 through December 1994. The patients were usually referred from industrial health care units or regional social insurance offices due to the presence of psychiatric or neurological symptoms and exposure to organic solvents. Only patients without other disorders of the central nervous system were included in the study. Women were few, and they were excluded for the sake of homogeneity.

The patients were subjected to a detailed symptom inquiry including a validated questionnaire (1) with focus on abnormal fatigue, deterioration of memory, personality changes of the aggressive or depressive type, frequency of headache, and sexual interest. The psychometric examination recommended by the Swedish Psychotechnical Institute (1) was performed for all the patients. Since air concentrations of solvents had been measured in the workplaces for only a minority of the patients, the intensity of exposure had to be indirectly estimated according to the frequencies and character of the acute solvent-related symptoms. Nor would it have been possible to account for long-term exposure by any other means than our general knowledge of exposure levels in various jobs. Thus the exposure of all the subjects was assessed from a thorough interview to identify the type of exposure and its duration and intensity.

The patients were classified into the three categories of solvent-induced chronic toxic encephalopathy (CTE), incipient CTE, and non-CTE. For the diagnosis of CTE, the following requirements had to be met: (i) relevant symptoms and (ii) presence of pathological findings in the psychometric function tests. For incipient CTE $(N=20)$ no clearly pathological results in the psychometric examination were present, but the symptoms of these subjects were essentially the same as for manifest CTE $(\mathrm{N}=21)$. For the non-CTE group $(\mathrm{N}=19)$, only vague and inadequate symptoms and no functional im- pairment in the psychometric examination were present. The mean age of the patients with CTE, incipient CTE, and non-CTE was 50, 52, and 45 years, respectively.

The duration of exposure to organic solvents ranged from 4 to 45 years and was seven years or more (mean 22 years) for the CTE patients. Incipient CTE patients had an exposure time of eight years or more (mean 20 years) and for non-CTE, the minimum exposure time was four years (mean 15 years). Moreover, all the subjects were classified into a solvent exposure intensity category from 2 to 5 on a scale of $0-5$, as used in an earlier study (5), where 0 indicates no exposure and 1 is extremely low exposure, eg, that of cleaners. Examples of occupations included in category 5 , the highest exposure group, were degreasers and floor layers. Construction painters and spray painters without proper protection against solvents appeared in category 4 . In category 3 were printers and repairmen. Artistic painters and aircraft motor assemblers are examples of occupations in category 2. An exposure index was created by multiplying the solvent intensity category by years of exposure, thereby obtaining a score from 12 to 180 . The median value was 60 points. Three persons with 60 points were included in the otherwise smallest group. Dividing the material at the median therefore resulted in 29 subjects in the low exposure group and 31 in the high exposure group.

DNA (deoxyribonucleic acid) was isolated from blood leukocytes by means of the Wizard Genome Purification kit (Promega Inc). GSTM1 genotype was determined in patient DNA using polymerase chain reaction (PCR) with primers specific to exon 4 and exon 5 of the GSTM1 (6). As an internal control for the amplification reaction, an additional primer was included which anneals to another class of the $\mu$ gene, GSTM4, producing a PCR (polymerase chain reaction) product with a different size (see figure 1) (6). All the PCR analyses were performed and scored blindly with regard to diagnosis.

\section{$\begin{array}{lllllll}1 & 2 & 3 & 4 & 5 & 6 & \mathrm{BI}\end{array}$}

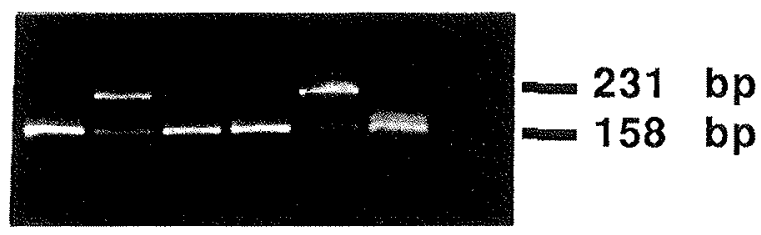

Figure 1. GSTM1 and GSTM4 gene, the polymerase chain reaction (PCR) products resolved by electrophoresis on $3 \%$ NuSieve $/ 1 \%$ agarose gel. A $10-\mu$ l aliquot of the reaction mixture was loaded. A 158 bp DNA fragment can be seen in all the PCR reactions and a 231 bp DNA fragment was only present in samples containing the GSTM1 gene. (lanes 2 and 5: GSTM1 positive genome; lanes 1,3,4, and 6: GSTM1 null genome (GSTM-), Bl: a negative control sample, ie, a PCR reaction with no DNA added) 


\section{Results}

Figure 1, lanes 2 and 5, shows the presence of a $231 \mathrm{bp}$ DNA fragment representing GSTM1 gene carriers and its absence in GSTM1- subjects, lanes 1,3,4, and 6. The 158 bp DNA fragment amplified from the GSTM4 gene in the same reaction tube was identified in all the subjects, excluding the possibility of a false interpretation due to failure in the amplification reaction. Table 1 shows the distribution of the GSTM1 genotypes detected among the CTE, incipient CTE, and non-CTE patients. The relative proportion (RP) of GSTM1 null genotypes in patients with diagnosed CTE compared with the non-CTE group was significantly increased $[\mathrm{RP}=2.55,95 \%$ confidence interval $(95 \% \mathrm{CI}) 1.0-6.2]$, whereas in the incipient CTE group the relative proportion for the GSTM1 null genotype was not significantly different from that of the non-CTE group. With regard to high or low solvent exposure, the GSTM1 null genotype appears to modify the risk of having CTE (ie, the absence of the gene amplifies the effect of solvent exposure) (table 2). There was an approximately eightfold risk of being affected by CTE in the high-exposure group if there was a lack of the GSTM1 gene, while heavy exposure to solvents increased the risk for CTE fourfold for subjects possessing the gene. When the CTE and incipient CTE groups were

Table 1. Relative proportion (RP) of the GSTM1 null genotype by degree of severity of chronic toxic encephalopathy (CTE) and solvent exposure. The proportion of occurrence of the GSTM1 gene in the non-CTE was used as the reference. $(95 \% \mathrm{Cl}=95 \%$ confidence interval)

\begin{tabular}{lrrrrr}
\hline $\begin{array}{l}\text { Patient } \\
\text { category }\end{array}$ & $\begin{array}{c}\text { GSTM1 } \\
\text { genotype }\end{array}$ & Total & RP & $95 \% \mathrm{Cl}$ \\
\cline { 2 - 3 } & + & - & & & \\
\hline Non-CTE & 11 & 8 & 19 & $(1.0)$ & $\cdot$ \\
Incipient CTE & 9 & 11 & 20 & 1.29 & $0.6-2.4$ \\
CTE & 4 & 17 & 21 & 2.55 & $1.0-6.2$ \\
\hline
\end{tabular}

a GSTM1+ designates a genotype in which the wild type alleles are retained and GSTM1-designates a genotype in which the wild type alleles are homozygously deleted. combined, the risks were lower but still more than doubled for those with high exposure and a lack of GSTM1 (table 2).

\section{Discussion}

The GSTM1- polymorphism has attracted much interest because it seems to be associated with an increased susceptibility to certain malignancies, but also to other diseases like biliary cirrhosis and hepatic diseases $(4,6-8)$. Most solvents are lipid soluble and affect the central nervous system after acute and chronic exposures, but there is no established mechanistic explanation for solvent-induced CTE. It has been hypothesized that reactive electrophiles formed during oxidative or reductive biotransformation pathways are toxic for nerve cells or that solvents or their metabolites influence membrane fluidity (9).

Glutathione S-transferases are among the most important enzyme systems to protect cellular structures from damage by reactive intermediates, and there are several isozymes participating in the deactivating reactions (2, 7-9). Little is known about the substrate specificity for the different glutathione S-transferase isozymes, but it seems as if the hydrophobic substrate binding site is rather nonspecific, whereas the binding pocket for reduced glutathione is highly specific $(10,11)$. GSTM1 has been considered to inactivate bulky reactive metabolites due to its efficient conjugation of benzo[a]pyrene-4,5-oxide, but it also displays high catalytic activity to smaller aromatic substrates like styrene7,8 -oxide (9). Thus it is plausible to assume the involvement of GSTM1 in the biotransformation of other solvent-derived and small substrates as well. This contention is supported in a Japanese study, in which an underrepresentation of persons expressing the GSTM1 enzyme was found among patients with alcoholic liver disease and liver carcinoma (25\% and $20 \%$, respectively) (12).

Table 2. Relative risks (RR) of CTE (chronic toxic encephalopathy) and incipient CTE in relation to the GSTM1 null (GSTM1-) genotype and solvent exposure. GSTM1+ subjects with low exposure were used as the reference.

\begin{tabular}{|c|c|c|c|c|c|c|c|}
\hline \multirow[t]{2}{*}{ Patient category } & \multirow[t]{2}{*}{ Non-CTE } & \multirow[t]{2}{*}{ Incipient CTE } & \multirow[t]{2}{*}{ CTE } & \multicolumn{2}{|c|}{ CTE/non-CTE } & \multicolumn{2}{|c|}{ CTE+incipient CTE/non-CTE } \\
\hline & & & & $\mathrm{RR}$ & $95 \% \mathrm{Cl}$ & $\mathrm{RR}$ & $95 \% \mathrm{Cl}$ \\
\hline \multicolumn{8}{|c|}{ Low exposure $(N=29)$} \\
\hline $\begin{array}{l}\text { GSTM1+c } \\
\text { GSTM1-d }\end{array}$ & $\begin{array}{l}8 \\
6\end{array}$ & $\begin{array}{l}4 \\
7\end{array}$ & $\begin{array}{l}1 \\
3\end{array}$ & $\begin{array}{l}1 \\
3.0\end{array}$ & 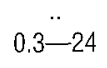 & $\begin{array}{l}1 \\
1.6\end{array}$ & $0.7-3.6$ \\
\hline \multicolumn{8}{|c|}{ High exposure $(N=31)$} \\
\hline $\begin{array}{l}\text { GSTM1+c } \\
\text { GSTM1- }\end{array}$ & $\begin{array}{l}3 \\
2\end{array}$ & $\begin{array}{l}5 \\
4\end{array}$ & $\begin{array}{r}3 \\
14\end{array}$ & $\begin{array}{l}4.5 \\
7.9\end{array}$ & $\begin{array}{l}0.6-34 \\
1.2-50\end{array}$ & $\begin{array}{l}1.9 \\
2.3\end{array}$ & $\begin{array}{l}0.8-4.1 \\
1.1-4.8\end{array}$ \\
\hline
\end{tabular}

a $\chi^{2}$ for linear trend (Mantel extension) $=4.99 ; P=0.025$.

$b \chi^{2}$ for linear trend (Mantel extension) $=1.89 ; P=0.169$

- GSTM1+ designates a genotype in which the wild type alleles are retained.

¿ GSTM1-designates a genotype in which the wild type alleles are homozygously deleted. 
This is the first study in which the influence of a certain genotype has been demonstrated to be associated with the development of CTE. The study is limited in size, and further investigations are needed to confirm our findings and to allow more definite conclusions. The basis for the observed increased susceptibility is unclear, but it is reasonable to believe that subjects with the GSTM1- genotype display less effective detoxification of reactive intermediates in the biotransformation and are therefore at increased risk when exposed. It is noteworthy in respect to CTE that GSTM1 enzyme activity is found in the brain (13); this result supports the role of GSTM1 as a protecting enzyme in this tissue. Our study also demonstrates the power of molecular genetic epidemiologic studies to find associations between exposures and disease (14), and it illustrates the interplay between environmental and genetic factors.

\section{References}

1. Axelson $\mathrm{O}$, Hogstedt $\mathrm{C}$. The health effects of solvents. In: Zenz C, Dickerson OB, Horvath EP, editors. Occupational medicine, 3rd edition. St Louis (MO): Mosby 1994:764-78.

2. Sies H, Ketterer B, editors. Glutathione conjugate and significance. London: Academic Press, 1988. CRC critical reviews in biochemistry and biology.

3. Mannervik B, Awasthi YC, Board PG, Hayes JD, Di Ilio C, Ketterer B, et al. Nomenclature for human glutathione transferases. Biochem J 1992;282:305-8.

4. Brockmöller J, Kerb R, Drakoulis N, Staffeldt B, Roots I.
Glutathione S-transferase M1 and its variants A and B as host factors of bladder cancer susceptibility: a case-control study. Cancer Res 1995;54:4103-11.

5. Flodin U, Fredriksson M, Persson B, Axelson O. Chronic lymphatic leukemia and engine exhausts, fresh wood and DDT: a case-referent study. Br J Ind Med 1988;45:33-8.

6. Zhong S, Wyllie AH, Barnes D, Wolf CR, Spurr NK. Relationship between the GSTM1 genetic polymorphism and susceptibility to bladder, breast and colon cancer. Carcinogenesis 1993;14:1821-24

7. Tsuchida S, Sato K. Glutathione transferases and cancer. Crit Rev Biochem Mol Biol 1992;27:337_84.

8. Hayes PC, Bouchier IAD, Beckett GJ. Glutathione S-transferase in humans in health and disease. Gut 1991;32:813-8.

9. Beckett GJ, Hayes JD. Glutathione S-transferases: biomedical applications. Adv Clin Chem 1993;30:281-380.

10. Mannervik B, Danielsson VH. Glutathione transferases structure and catalytic activity. CRC Crit Rev Biochem 1988; 23:283-337.

11. Wilce MCJ, Parker MW. Structure and function of glutathione S-transferases. Biochim Biophys Acta 1994;1205:1-18.

12. Harada S, Abei M, Tanaka N, Agarwai DP, Goedde HW Liver glutathione S-transferase polymorphism in Japanese and its pharmacogenetic importance. Hum Genet 1978;75:3225 .

13. Strange RC, Fryer AA, Matharoo B, Zhao L, Broome J, Campbell DA, et al. The human glutathione S-transferases: comparison of isoenzyme expression in normal and astrocytoma brain. Biochim Biophys Acta 1992;1139;222-8.

14. Söderkvist P, Axelson O. On the use of molecular biology data in occupational and environmental epidemiology. J Occup Environ Med 1995;37:84-90.

Received for publication: 11 December 1995 